\title{
Heart Failure Detection Using Quantum-Enhanced Machine Learning and Traditional Machine Learning Techniques for Internet of Artificially Intelligent Medical Things
}

\author{
Yogesh Kumar $\mathbb{D}^{1},{ }^{1}$ Apeksha Koul, ${ }^{2}$ Pushpendra Singh Sisodia, ${ }^{1}$ Jana Shafi $\mathbb{D}^{3}$ \\ Kavita Verma, ${ }^{4}$ Mehdi Gheisari $\left(\mathbb{D},{ }^{5}\right.$ and Mohamad Bagher Davoodi ${ }^{6}$ \\ ${ }^{1}$ Indus Institute of Technology \& Engineering, Indus University, Ahmedabad 382115, India \\ ${ }^{2}$ Department of Computer Engineering, Punjabi University, Patiala 147002, India \\ ${ }^{3}$ Department of Computer Science, College of Arts and Science, Prince Sattam bin Abdul Aziz University, \\ Wadi Ad-Dwasir 11991, Saudi Arabia \\ ${ }^{4}$ Department of Computer Science and Engineering, Chandigarh University, Mohali 140413, India \\ ${ }^{5}$ Young Researchers and Elite Club, Parand Branch, Islamic Azad University, Parand, Iran \\ ${ }^{6}$ Bounty Company, Iran \\ Correspondence should be addressed to Mehdi Gheisari; mehdi.gheisari61@gmail.com
}

Received 10 October 2021; Revised 30 October 2021; Accepted 1 December 2021; Published 17 December 2021

Academic Editor: Mohammad R Khosravi

Copyright ( 92021 Yogesh Kumar et al. This is an open access article distributed under the Creative Commons Attribution License, which permits unrestricted use, distribution, and reproduction in any medium, provided the original work is properly cited.

\begin{abstract}
Quantum-enhanced machine learning plays a vital role in healthcare because of its robust application concerning current research scenarios, the growth of novel medical trials, patient information and record management, procurement of chronic disease detection, and many more. Due to this reason, the healthcare industry is applying quantum computing to sustain patientoriented attention to healthcare patrons. The present work summarized the recent research progress in quantum-enhanced machine learning and its significance in heart failure detection on a dataset of 14 attributes. In this paper, the number of qubits in terms of the features of heart failure data is normalized by using min-max, PCA, and standard scalar, and further, has been optimized using the pipelining technique. The current work verifies that quantum-enhanced machine learning algorithms such as quantum random forest (QRF), quantum $K$ nearest neighbour (QKNN), quantum decision tree (QDT), and quantum Gaussian Naïve Bayes (QGNB) are better than traditional machine learning algorithms in heart failure detection. The best accuracy rate is (0.89), which the quantum random forest classifier attained. In addition to this, the quantum random forest classifier also incurred the best results in F1 score, recall and, precision by (0.88), (0.93), and (0.89), respectively. The computation time taken by traditional and quantum-enhanced machine learning algorithms has also been compared where the quantum random forest has the least execution time by 150 microseconds. Hence, the work provides a way to quantify the differences between standard and quantum-enhanced machine learning algorithms to select the optimal method for detecting heart failure.
\end{abstract}

\section{Introduction}

Quantum computing is a revolutionary concept based on the fundamental principles of nature, i.e., quantum mechanics. With the advancements in physics in the early twentieth century, methods of observation and purity of materials reached the level at which some quantum phenomena became detectable [1], such as regular transistors present in every modern computer or device. It is operated by directing large clouds of carriers of electrical current using engineered materials and quantum-based principles (band structure, localized states, etc.). They produce unusual behaviour for naturally found materials-an ability to precisely control current with current, or current via light, or light via current [2]. Quantum-enhanced machine learning is at the junction of both current research areas: quantum 
computing and machine learning. It prospects the interaction between machine learning and quantum computing to inquire how outcomes and results of approaches from one area can be utilized to compute the issues of the other field. With a burgeoning amount of data, current machine learning systems rapidly intend to confine classical computational models. In this sense, quantum computational power can offer an advantage in machine learning tasks [3]. Currently, quantum technology is divided into three distinct fields: quantum computing, quantum information, and quantum cryptography. Quantum computation exerts its effect due to the generous permutations that make quantum computers twice as fast as memory fills up with the addition of each qubit. As a result, we require $N$-bits of binary numbers to describe the $N$-bits classical bit system. Recent research breakthroughs have made significant contributions to the advancement of machine learning algorithms by using the benefits of quantum computing. However, tremendous work has been done to design and implement quantum versions [4] of ANN. Besides this, they are also based on more natural aspects that are yet to be accomplished [5]. Some authors tried to develop a complete quantum algorithm that could achieve pattern recognition problems [6]. In contrast, others suggested implementing subprograms of traditional machine learning algorithms on a quantum system. The adiabatic quantum-enhanced machine learning method appears to be applicable to some kinds of optimization problems $[7,8]$.

Despite such tremendous advancements in the medical domain, heart failure has posed an immense threat to changing patients' health. Recent years have witnessed a considerable increase in mortality and morbidity due to heart failure threat to lives [9]. It challenges healthcare providers as it is leading to tremendously high rates of mortality and morbidity. It results from the defects in the myocardium, which further results in the ejection of blood or impairment of ventricular filling. Based on the location, heart failure (HF) can be classified as biventricular left ventricular or right ventricular. It can also be classified as chronic or acute. It is found that females and aged people suffer from HFpEF (heart failure with preserved ejection fraction) [10]. The significant symptoms of heart failure include shortness of dyspnea, orthopnea, nocturnal dyspnea, lethargicness, pedal edema, tachycardia, jugular venous pressure, and S3 gallop [5].

Thus, traditional machine learning and quantumenhanced machine learning algorithms in healthcare are extensively used to aid the patients and medical staff in many diverse ways. These algorithms have been used for heart failure imaging applications, the contribution of its risk evaluation in different ways, and predicting heart failure detection. Researchers deployed Rubidium-based quantum sensors in one research [7] to detect signals made by atrial fibrillation, a disease that creates an unpredictable and abnormally high heartbeat. They had seen an array of their quantum sensors that can be positioned over the heart for supplying primary data. They also suggested that quantum technologies can increase the clinical results of atrial fibrillation.
In addition to this, the Internet of Medical Things (IoMT) has also been considered to be the wave of the future in the field of healthcare. It is referred to as a collection of medical devices and apps connected to healthcare systems via online computer networks [11]. The Internet of Medical Things (IoMT) comprises smart devices, such as wearables and medical monitors, that are created for healthcare reasons and may be utilized on the human body, at home, in the community, and clinical settings, among other places. Numerous healthcare providers are adopting IoMT apps to enhance treatments and illness management, minimize mistakes, improve patient experience, manage medications, and lower costs [12]. In addition to this, IoMT also reduces the number of needless hospitalizations and the total load on healthcare systems by linking patients directly to their physicians and facilitating the transfer of medical data through a secure network instead of traditional methods. According to a new assessment by Deloitte, its influence on the healthcare business is evident and permanent as it is predicted to increase from $\$ 41$ billion in 2017 to $\$ 158$ billion by 2022 . Hence, in a nut shell, advancements in wireless communications, sensor networks, mobile devices, big data analysis, and cloud computing, the Internet of Medical Things (IoMT) is transforming the healthcare industry by delivering targeted and personalized medicine and also enable seamless communication of medical data between healthcare providers and patients.

Therefore, it can be said that traditional and quantumenhanced machine learning algorithms for the Internet of Medical Things (IoMT) aids in making an earlier diagnosis of diseases and identifies predictive characteristics in different pathologic conditions.

To assess where and how traditional and quantumenhanced machine learning techniques may provide opportunities for detection of heart failure, it is essential to understand the use and applicability of the different techniques such as QKNN, quantum Naïve Bayes, quantum decision tree, and quantum random forest. The primary concern of the presented paper is to highlight the usage and efficiency of the traditional and quantum-enhanced machine learning algorithms along with its comparative analysis based on accuracy, F1-score, recall, and precision by using scalar (min-max, standard, and PCA) and pipelining technique.

1.1. Contribution. Quantum computers are based on quantum physics, which allows them to operate far faster than conventional computers without requiring massive hardware systems. Thus, quantum computing can assist computers in achieving faster processing speeds and overcoming more composite problems. This paper puts forward a systematic and experimental study on quantumenhanced machine learning and its utilization in different research areas. In this article, implemented work has been done to detect heart failure using various attributes with the help of traditional and quantum-enhanced machine learning algorithms. Moreover, we have also shown the comparison between them to have the best algorithm with the highest accuracy rate, $F 1$ score, recall, precision, and less computation time. The significant intention of the paper is 
to highlight the importance of quantum-enhanced machine learning in the healthcare sector. The contributions of the article are as follows:

(1) It provides an inclusive knowledge of quantumenhanced machine learning

(2) It walks through recent trends and existing applications and systems based on quantum computing

(3) A detailed study of applications of quantumenhanced machine learning in the healthcare domain is presented

(4) It also shows the implementation of quantumenhanced machine learning algorithms for the detection of heart failure and compared it with the traditional machine learning algorithms for different parameters such as accuracy, precision, recall, and F1-score using a different scale and transformation techniques such as min-max, standard, PCA, and other pipelining technique to optimize the results

\section{Process of Traditional Machine Learning and Quantum-Enhanced Machine Learning}

The core of machine learning is to train the machine using algorithms that have been executed to handle the data. The traditional methods of machine learning, via its subsets of supervised and unsupervised learning, i.e., deep learning, helps in classifying the images and identifying the patterns and speech, and controls big data, etc. [13]. Nowadays, it becomes necessary to have new approaches to manage, organize, and classify the diverse range of available data [14].

The long-established machine learning has received much heed and investments from different organizations and industries [15]. The industries whose labor is involved in effective data warehouse management are capable of handling a diverse range of data and are very keen to know new approaches to executing this. Quantum-enhanced machine learning is a promising solution that can be used to eradicate such limitations [16]. One of the problems to be resolved in quantum-enhanced machine learning is the vulnerability available in the input data that the proposed methodologies can treat and handle effectively [17]. The critical principle that differentiates quantum-enhanced machine learning in traditional algorithms is how the learning takes place on different data. Unlike conventional algorithms, which mainly focus on design, quantum algorithms are versatile and can effectively solve different problems. Adaptive learning autonomously finds the possible set of behaviors and patterns to solve a complex problem [18].

Although traditional machine learning [19] has been observed to be a flexible and adaptive procedure that can effectively map different patterns, some complex problems still exist that cannot be efficiently solved by the conventional method of machine learning algorithms. Comparing regular machine learning and quantum-enhanced machine learning processes is depicted in Figure 1, which compares

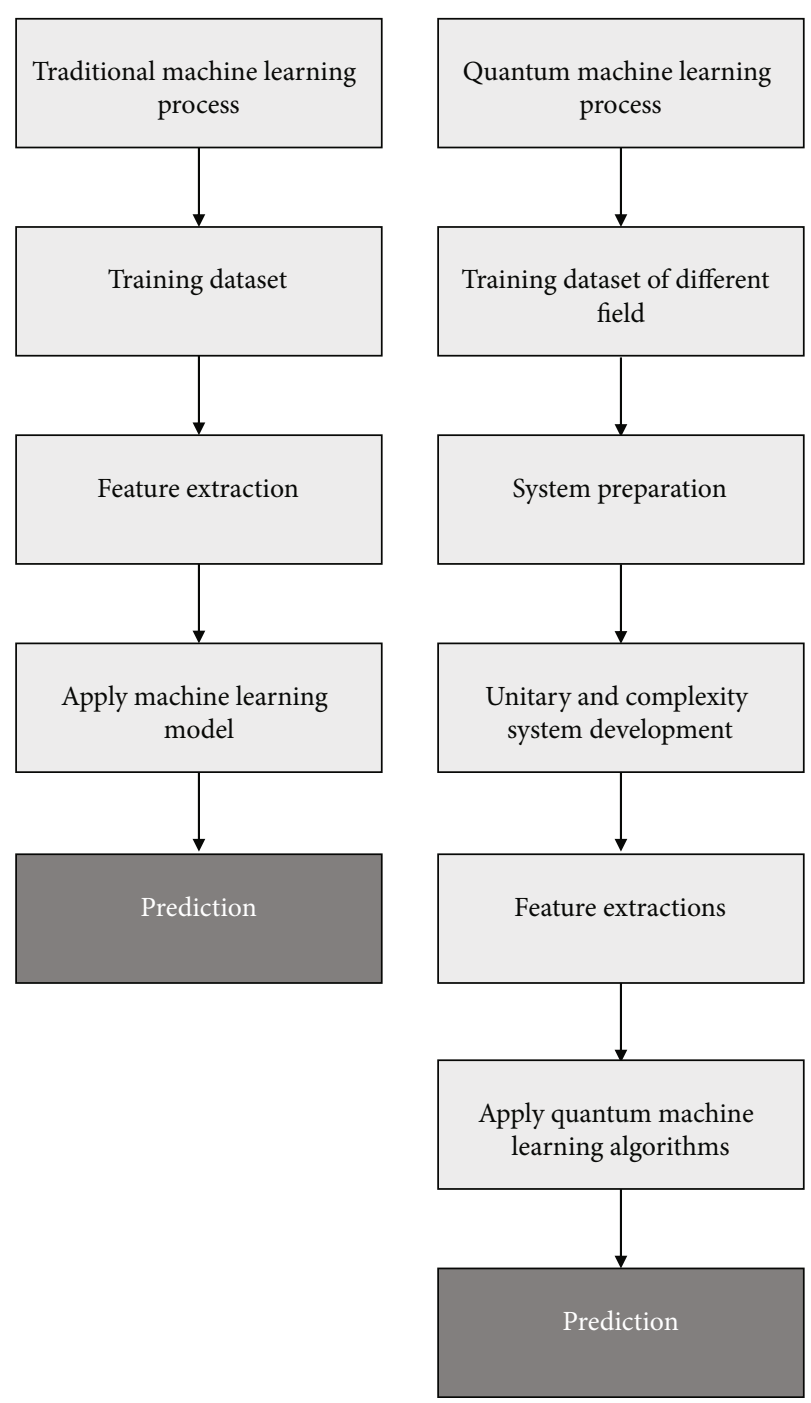

Figure 1: Traditional machine learning and quantum-enhanced machine learning process.

the two methods. In the case of traditional machine learning, features are extracted, and then, machine learning algorithms are applied to predict the output. However, in the case of quantum machine learning, the data is subjected to prepare the model where a unitary and complexity system is developed. Then, features are extracted, after which quantum-enhanced machine learning algorithms are applied to predict the outcome. Therefore, the companies whose labor is involved in large record storage organizations are very interested in learning new approaches. Hence, the quantum-enhanced machine learning domain is found to be one of these promising approaches. However, the interest to implement these techniques through quantum computation paves the way to quantum-enhanced machine learning [20].

2.1. Working of Quantum-Enhanced Machine Learning. Quantum-enhanced machine learning is a technique developed to augment the algorithms used in regular machine learning. Quantum-enhanced machine learning is a subfield 
of quantum information processing research [3] that focuses on developing machine learning algorithms capable of learning from data. Quantum computers compute information using the principles of quantum theory, and quantum algorithms are a collection of assertions that execute on these systems [20]. Many quantum algorithms have been designed for machine learning techniques such as neural networks and graphical models. Quantum-enhanced machine learning algorithms mostly rely on the Grover search, which speeds up unordered datasets [21]. Many quantumenhanced machine learning-based algorithms have been developed for pattern recognition and data extraction [22]. Quantum-enhanced machine learning-based algorithms use quantum computing to solve complex issues for ordinary machine learning algorithms and are executed by acclimatizing established procedures to implement on a potential quantum system [23]. With this pace of advancement, it can be observed that such machines are rapidly used for applications in the coming era, which will then ease the process of analyzing universal information. The rising field also incorporates techniques, namely, robust machine learning methods, that can extend quantum information theory [24]. Quantum-enhanced machine learning is optimizing traditional artificial intelligence systems and is considered one of the future research areas in using machine and deep learning algorithms, as stated in Table 1.

\subsection{Role and Platforms of Quantum-Enhanced Machine} Learning in Healthcare. Quantum-enhanced machine learning is accelerating its growth and adoption due to its immense potential. Compared to traditional machine learning algorithms, quantum-enhanced machine learning techniques can reduce training time, handle complex network topology, automatically adjust network hyperparameters, perform complex matrix and tensor manipulation at high speeds, and use quantum tunneling to achieve actual objective function goals.

As far as the healthcare industry is concerned, it is using quantum computations to carry a patient-centric concern for medical care clients. Quantum computing and healthcare systems enable hardware solutions that can significantly benefit the healthcare industry in evaluating and treating complicated medical situations [36]. Quantum computing imbues the digital world with quantum physics, enabling computers to process data quicker and solve more complex issues [37]. Additionally, there are several critical concepts associated with quantum computing, including quantum bits (qubits), which are information units that can exist in either an ON or an OFF state, quantum superposition, which allows particles to exist in multiple states and provides tremendous power and flexibility for solving complex problems, entanglement, which occurs when pairs or groups of particles are generated, interact, or share spatial proximity, tunneling in which a particle goes through what appears to be an energy barrier, and quantum gate that acts on a collection of quantum states called basis states to produce the desired output state.

Machine learning and artificial intelligence are being used with traditional computing resources to interpret CT scans, aid surgical procedures, and analyze big data to develop predictive models of disease [38]. Quantum computing holds no value to medicine without the parallel increase in the availability of clinically meaningful data from numerous sources [9]. Parsons18 [39] predicted that when the first quantum computing devices became operational in roughly 2000, quantum computing applications would shape the future of medical imaging. Quantum computing will be used to analyze diagnostic pictures through the use of artificial intelligence. Not only will picture detail be exponentially increased but physician's interpretation of data will be assisted since effective machine learning can train a quantum computer to detect abnormal discoveries with more precision than the human eye. Second, quantum computing will aid in the development of more effective cancer medicines. Computers are now utilized to manage the myriad of variables involved in developing a radiation plan targeting cancer cells while sparing healthy cells. Machine learning and artificial intelligence have been combined with traditional computer resources to help interpret CT scans, surgical operations, and the analysis of large amounts of data to construct illness prognostic models. Quantum computing is of little use to medicine unless it is accompanied by an increase in the availability of clinically valuable data from various sources. The challenges associated with implementing a healthcare system in which quantum computing analyzes decades of data from billions of clinical encounters and recommends a specific medication to a patient presenting with new-onset depression, cancer, diabetes, or any other condition will be overcome when data from multiple sources, such as existing patient data networks, biobanks, and wearable health devices, are combined.

Thus, quantum computing uses decades of data from billions of clinical encounters and recommends a specific medication for patients presenting with new-onset depression, cancer, diabetes, etc. [40]. Quantum computing can aid in the provision of precise medical imaging and medicines. There are a variety of applications for quantumenhanced machine learning algorithms, including diagnostic aid, where quantum computing has the potential to improve image analysis, including processing stages such as edge identification and picture matching. Care providers may enhance diagnoses while eliminating the need for recurrent intrusive diagnostic testing, a process known as precision medicine. Precision medicine attempts to customize preventative and treatment methods to the person [41]. Quantum-enhanced machine learning techniques enable earlier, more accurate, and granular risk forecasting, pricing in which quantum algorithms may help superior classification and pattern detection, thereby aiding in discovering abnormal behavior and eliminating fraudulent medical claims. Additionally, in addition to determining the optimal medication, quantum-enhanced machine learning can benefit healthcare systems in a variety of other ways, including the following:

(a) Quantum imaging equipment can provide exact images, enabling the observation of single molecules [14] 


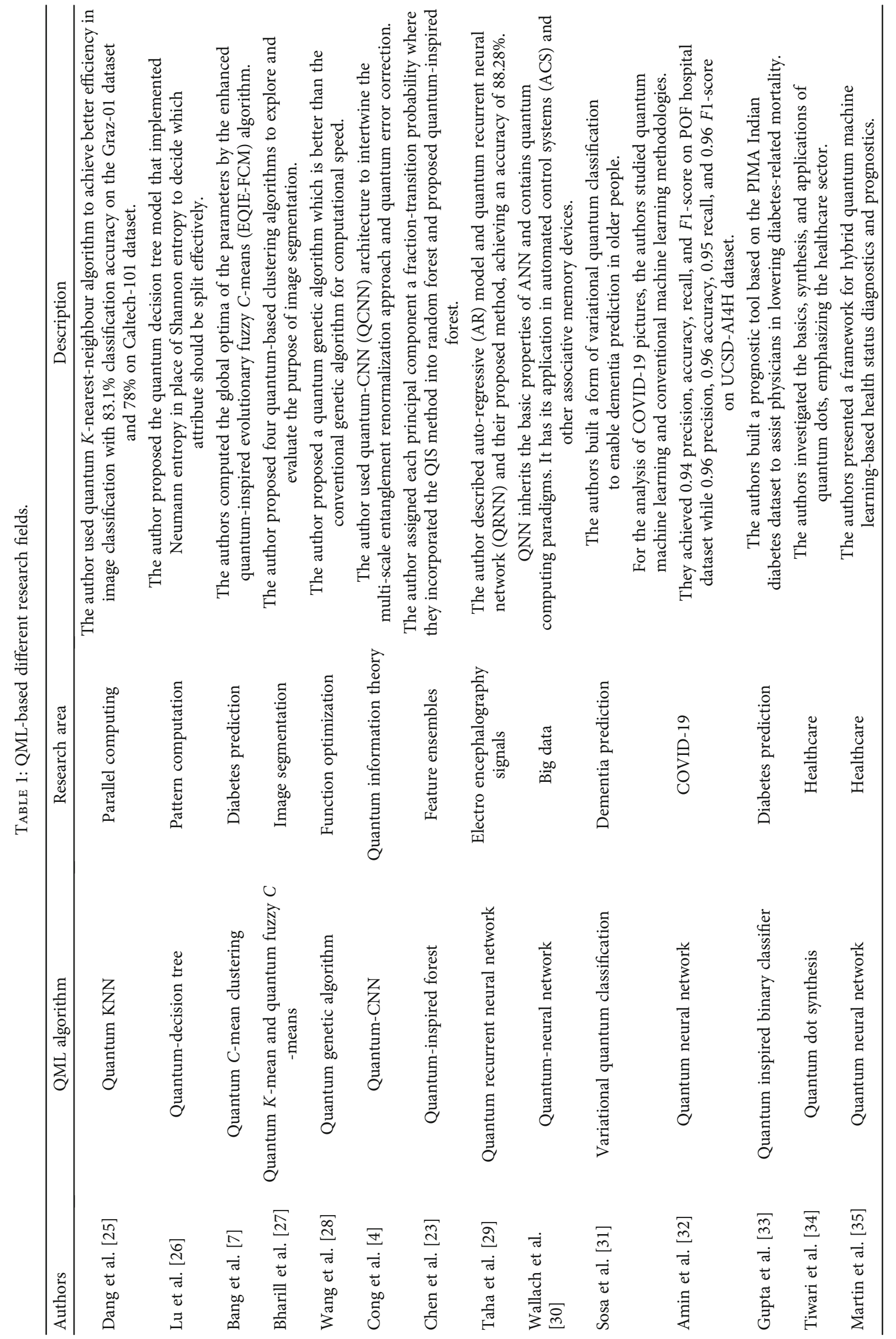


(b) When combined with quantum computing, machine learning algorithms can assist physicians in understanding therapy outcomes

(c) Machine learning is used to detect irregularities in the human body, while quantum computing aids in the interpretation of therapy outcomes [42]

(d) Radiation beams are utilized in quantum-enhanced machine learning to kill or halt the proliferation of damaged cells [43]

(e) Quantum computers enable clinicians to discover the optimal therapy for each simulation [10]

(f) The capacity of quantum computing to process algorithms has drawn the attention of administrators from a variety of businesses. According to one estimate, the quantum computing business was worth \$93 million in 2019 and is expected to grow to $\$ 283$ million by 2024 [44]

(g) The potential for quantum computing to identify treatments targeting specific forms of cancer significantly adds to its rise in the healthcare business. The amount of money spent on the research and development team, the amount of time spent on research, and the time required for complete radiation analysis will decrease if quantum computing and healthcare systems integrate [13]

Table 2 illustrates the different quantum-enhanced machine learning platforms and their purpose in the healthcare domain, along with their utility of in healthcare.

\section{Materials and Methods}

This section of the article deals with implementing traditional machine learning algorithms and includes information regarding the dataset considered, and the approaches followed.

3.1. Dataset Description. This section of the paper discusses the dataset that has been considered for detecting heart failure using traditional machine learning and quantumenhanced machine learning algorithms. The following are the details:

(1) The dataset considered has been collected from the UCI repository

(2) It contains 304 instances with 14 attributes like age, sex, blood pressure, angina, cholesterol, blood sugar, ECG, and maximum heart rate, as shown in Table 3

(3) The target variable is the diseased event to check whether the patient is suffering from heart failure or not

(4) There are no missing values in the considered dataset
Machine learning techniques have proved their robustness in every domain. Thus, we decided to detect heart failure using traditional and quantum-enhanced machine learning algorithms to help medical professionals. Table 3 describes 14 attributes present in the dataset.

The different attributes of the health issues are age which is mainly a significant dangerous cause in budding heart failure. It has been approximated that 82 per cent of the folks who belong to 65 and older are more prone to heart failure. At the same time, the threat of stroke gets doubled every decade after attaining age 55. The second attribute is sex, where males are at higher threat of coronary ailment than premenopausal women. However, if a woman has diabetes, she can have more threat of heart disease than a male. The other factors are chest pain, also known as angina, and is caused when enough oxygen-rich blood does not reach the heart's muscles. The heart patient may feel as if someone is squeezing their chest. The uneasiness can also arise in the shoulders, arms, neck, jaw, or back. The person might also feel digestion issues.

The increased amount of low-density lipoprotein cholesterol, also known as serum cholesterol, on the other hand, is likely to be the cause of arteries collapsing. Uncontrolled blood pressure has the potential to harm the arteries that supply our hearts. When high blood pressure is combined with additional factors like obesity, high cholesterol, or diabetes, the risk of heart failure increases even more. The risk of cardiac arrest is also increased when triglycerides are high. High levels of high-density lipoprotein cholesterol, on the other hand, reduce the risk of a heart attack. A spike in blood sugar levels in the body is another cause of heart failure, which occurs when the pancreas does not generate enough hormones or respond to insulin.

Likewise, achieving maximum cardiac rate is also the cause of heart failure, where the rise in heart failure threat is linked with the escalation of heart rate. For example, it has been observed that the rise in cardiac rate by $10 \mathrm{bpm}$ raises the threat of heart failure by at least $20 \%$. The other factors responsible for heart attack are exercise-induced angina and peak exercise ST-segment. Angina-related discomfort might feel tight, gripping, or squeezing and can range from mild to severe. On the other hand, peak exercise is a treadmill ECG stress test in which pressure is recorded irregularly at a straight or downhill incline when the STsegment depression is higher than or equal to $1 \mathrm{~mm}$ at 60 $80 \mathrm{~ms}$.

3.2. Framework. This section of the article discusses the approach followed to functioning traditional and quantumenhanced machine learning algorithms.

Figure 2 shows the flow that has been followed for the process and its steps are as follows:

Step 1. The dataset was collected from the UCI repository, and its details have already been discussed in the dataset description section. 
TABLE 2: QML-based healthcare platforms [1, 7-9, 23, 38, 45, 46].

\begin{tabular}{|c|c|}
\hline $\begin{array}{l}\text { Developed } \\
\text { platform }\end{array}$ & Purpose \\
\hline PathAI & $\begin{array}{l}\text { They developed a machine-learning algorithm to } \\
\text { assist pathologists in making precise diagnoses. }\end{array}$ \\
\hline Enlitic & $\begin{array}{l}\text { Artificial intelligence deep learning for actionable } \\
\text { insights }\end{array}$ \\
\hline Freenome & Earlier cancer detection with AI \\
\hline $\begin{array}{l}\text { BioXcel } \\
\text { Therapeutics }\end{array}$ & $\begin{array}{l}\text { BioXcel Therapeutics employs AI to re-innovate } \\
\text { clinically approved products and medicines. }\end{array}$ \\
\hline BERG Health & Treating rare disease with AI \\
\hline XtalPi & $\begin{array}{c}\text { Combining AI-cloud and quantum computing- } \\
\text { based digital drug discovery }\end{array}$ \\
\hline
\end{tabular}

Atomwise

Deep

Genomics

BenevolentAI

Olive

Qventus

Babylon

Health

CloudMedX

For a better patient journey

Vicarious

surgical

Auris health

AI robots revolutionizing endoscopy

Intuitive

Pioneering robotic surgery

Microsure

Neural network for clinical trials

Identifying more promising prospects for developing medicines

Deep learning for targeted treatment

Automating the most repetitive processes in healthcare

Real-time patient flow optimization

Increasing access to healthcare

Virtual reality-enabled robotics for surgery

Step 2. The dataset obtained was preprocessed, which included checking for NULL values and converting string values to float (because string values cannot be directly used in machine learning algorithms), which was not applicable.

Step 3. For implementation, various libraries like time, copy, math, NumPy, matplotlib, sklearn, and most importantly, qiskit for quantum have been used.
How to use and also, it helps minimize errors in cancer detection.

It is used to streamline radiology diagnosis and analyses raw and unlabelled medical data such as radiology images and genomics.

Freenome is subjected to discover cancer in its early phases and subsequently helps build new methods of treating cancer.

BioXcel Therapeutics' mission is to reinvent drugs in the immunooncology and neuroscience domains. AI is believed to be the only instrument to maximize the value of clinically approved treatments and goods.

BERG is an AI-based clinical platform that leverages the treatment by quick mapping of disease, thereby helping develop medicines.

XtalPi's ID4 platform is an AI-based engine that predicts the chemical and pharmaceutical properties for reinventing drug design and development.

Atomwise uses AI technology to configure the most dangerous diseases such as Ebola and multiple sclerosis (MS).

Deep Genomics is a platform primarily based on AI that can develop drug-related disorders such as neuromuscular and neurodegenerative.

Benevolent is subjected to providing the appropriate treatment to the right patients at the required time by using AI for a better target. Also, it gives unearthed insights with the help of deep learning.

Olive is an AI-based platform built to automate the repetitive task of the healthcare industry and thus saves the precious time of administrators, thereby fostering them to accomplish higher-order tasks.

Qventus is a platform that is based on artificial intelligence, which aids in overcoming operational challenges primarily related to emergency rooms and patient safety.

Babylon is an artificial intelligence-based platform that enables patients to access various healthcare-related services via a powerful interactive interface.

CloudMedX provides AI tools to ease the burden by reducing manual work. It works by ingesting structured and unstructured data and then uses this data to get insights by using machine learning algorithms.

Vicarious Surgical aims to improve patient outcomes by fusing virtual reality with robots exhibiting AI technology.

Auris Health is leveraging medical intervention by introducing the Monarch platform. This platform integrates data science and microinstrumentation to bring improvement in endoscopies design and tools.

Intuitive's da Vinci platforms have come up with a pioneering robotic surgery technique in industries.

Microsure robots were designed with the ability to aid surgeons with robotic assistance and thereby to overcome human physical limitations.
Step 4. Then, standardization and transformation of the data have been done with the help of min-max scalar, standard scalar, and PCA scalar to scale all the features. Further, normalization and optimization of the data have been done to obtain better results using the pipelining technique.

Step 5. After this, the data was divided into two parts: training and testing set in the ratio of $8: 2$. The training data were 
TABLE 3: Description of dataset attributes.

\begin{tabular}{|c|c|c|}
\hline Attribute & Description & Input \\
\hline Age & Age of the patients & Years \\
\hline Sex & Sex $(1=$ male; $0=$ female $)$ & Float \\
\hline Trestbps & Resting blood pressure (in mmHg on admission to the hospital) & $\mathrm{mm} / \mathrm{Hg}$ \\
\hline $\mathrm{CP}$ & $\begin{array}{c}\text { Chest pain type- value 1: typical angina - value } 2 \text { : atypical angina - value } 3 \text { : nonanginal pain - value } 4: \\
\text { asymptomatic }\end{array}$ & Float \\
\hline Cholesterol & Serum cholesterol & $\mathrm{mg} / \mathrm{dl}$ \\
\hline Fps & (fasting blood sugar $>120 \mathrm{mg} / \mathrm{dl})(1=$ true; $0=$ false $)$ & Float \\
\hline Restecg & $\begin{array}{l}\text { Resting electrocardiographic results- value } 0 \text { : normal - value } 1 \text { : having ST-T wave abnormality (T wave } \\
\text { inversions and/or ST elevation or depression of }>0.05 \mathrm{mV} \text { ) - value } 2 \text { : showing probable or definite left } \\
\text { ventricular hypertrophy by Estes' criteria }\end{array}$ & Float \\
\hline Thalach & Maximum heart rate achieved & Binary \\
\hline Exang & Exercise induced angina $(1=$ yes; $0=$ no $)$ & Int \\
\hline Old peak & ST depression induced by exercise relative to rest & Continuous \\
\hline Slope & $\begin{array}{l}\text { The slope of the peak exercise ST segment- value 1: upsloping - value 2: flat - value 3: downsloping number of } \\
\text { major vessels }(0-3) \text { colored by flourosopy }\end{array}$ & Float \\
\hline CA & Follow up period number of major vessels $(0-3)$ colored by flourosopy & Float \\
\hline Thal & $3=$ normal; $6=$ fixed defect; $7=$ reversible defect & Float \\
\hline Target & $\begin{array}{l}\text { Whether person suffering through heart disease or not } \\
\qquad \begin{array}{l}0=\text { Normal } \\
1=\text { suffering }\end{array}\end{array}$ & Float \\
\hline
\end{tabular}

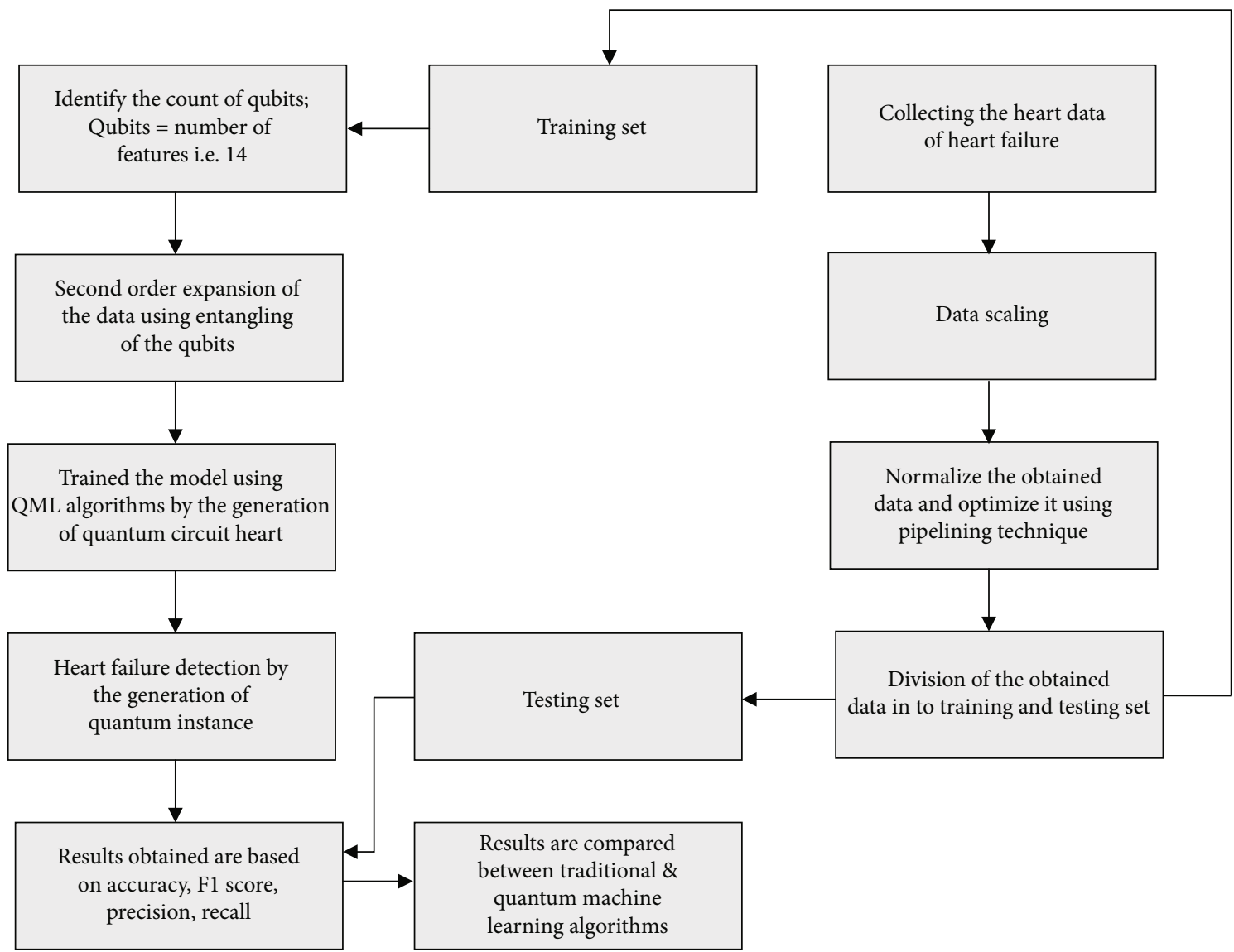

Figure 2: Proposed system design. 
used in developing the quantum-enhanced machine learning model.

Step 6. The number of qubits were specified, and the thumb rule to do so is as stated: number of qubits = number of features. So, for our dataset, the numbers of qubits are 14 .

Step 7. After this, the mapping of the features is done with the help of entangling qubits to the second-order expansion.

Step 8. Later, the circuit is generated for quantum-enhanced machine learning algorithms in which quantum instances for heart failure detection are formed for the implementation purpose. The model is trained now, and with the help of testing data, the results are obtained using various quality metrics such as accuracy, precision, recall, and F1-score.

3.3. Applied Algorithms for Heart Failure Detection. Incorporating quantum algorithms within the machine learning process gives rise to quantum-enhanced machine learning. It commonly uses machine learning algorithms to examine traditional data executed on a quantum processor [47]. Traditional machine learning techniques include the random forest classifier (random decision forests), an ensemble learning approach for classification, regression, and other applications. Using a randomly selected portion of the training data, the random forest classifier generates a series of decision trees. It may be the most popular and widely used AI computation based on its fantastic or spectacular display throughout a vast grouping scope [40]. Random forest algorithm is solved by

$$
\begin{gathered}
\operatorname{RFfi}_{i}=\frac{\sum_{j \in \text { all trees }} \text { normfi }_{i j}}{T}, \\
\text { normfi }_{i}=\frac{\mathrm{fi}_{i}}{\sum_{j \in \text { all features }} \mathrm{fi}_{j}}, \\
\mathrm{fi}_{i}=\frac{\sum_{j \text { :node } j \text { splits on feature } i} \mathrm{ni}_{j}}{\sum_{k \in \text { all nodes }} \mathrm{ni}_{k}}, \\
\sim \\
\mathrm{ni}_{j}=W_{j} C_{j}-W_{\text {left }(j)} C_{\text {left }(j)}-W_{\text {right }(j)} C_{\operatorname{right}(j)} .
\end{gathered}
$$

Here, $n i_{j}$ means importance of node $j, W_{j}=$ weighted number of samples reaching node $j, C_{j}=$ the impurity value of node $j$, left $(j)=$ child node from left split on node $j$, $\operatorname{right}(j)=$ child node from right split on node $j, \mathrm{fi}_{i}=$ the importance of feature $i, \mathrm{RFfi}_{i}=$ the importance of feature $i$ calculated from all trees in the random forest model, normfi $i_{i}=$ the normalized feature importance for $i$ in tree $j$, $T=$ total number of trees. $K$ nearest-neighbor classifier is a nonparametric AI strategy that is utilized for characterization just as relapse. It is based on the distances between a question and every model in the information by choosing the predetermined number nearest to the inquiry [48]. It is calculated by using the Euclidean Distance formula, which is shown as

$$
d(p, q)=\sqrt{\sum_{i=1}^{n}\left(q_{i}-p_{i}\right)^{2}}
$$

where $q$ and $p$ points are used to calculate the distance at $n$ different positions.

Likewise, there is a choice tree classifier, one of the regulated learning strategies and prescient demonstrating approaches utilized in insights, information mining, and AI. These are among the most mainstream AI calculations to provide their coherence and effortlessness [49]. Decision tree constructs characterization or relapse models as a tree structure. It divides an informative index into smaller subsets, resulting in a tree with choice hubs and leaf hubs as the final result. Decision tree are great instruments for assisting us with picking between a few blueprints [17]. It is calculated by using information gain (IG) and Gini index as shown in

$\mathrm{IG}=\operatorname{Entropy}(\mathrm{s})-[($ Weighted Avg $) * \operatorname{Entropy}($ each feature $)]$,

Entropy $($ s $)=-P($ yes $) \log _{2} P($ yes $)-P($ no $) \log _{2} P($ no $)$,

where $s=$ total number of samples, $P($ yes $)=$ probability of yes, and $P($ no $)=$ probability of no,

$$
\text { Gini Index }=1-\sum_{j} \mathrm{P}_{j}^{2}
$$

where $j$ denotes the number of features.

At the end, there is Naïve Baye's classifier which is a set of classifier algorithm that relies on Baye's theorem. It is used to calculate the conditional probability of a hypothesis being true given that order of information is also true [36]. Equation (9) is as follows

$$
P\left(H_{i} \mid D\right)=\frac{P\left(H_{i}\right) P\left(D \mid H_{i}\right)}{\sum_{j} P\left(H_{i}\right)},
$$

where $P\left(H_{i} \mid D\right)$ is the posterior probability, $P\left(H_{i}\right)$ is the likelihood, $P\left(H_{i}\right)$ is the class prior probability, and $P\left(H_{i}\right)$ is the posterior prior probability. On the other hand, there are quantum-enhanced machine learning algorithms used in quantum-enhanced machine learning software as part of a larger implementation such as a quantum random forest classifier that uses quantum trees to select the class and generate random number generators [50]. In quantum $K$ -nearest neighbour, the centroid is detected using the swap gates test between two states of the qubit. Assume that a training set $\boldsymbol{\tau}$ of feature vectors with their associated classifications is provided, together with an unclassified input vector $\vec{x}$, to select the class $c^{x}$ for the new input that matches most of its $k$ nearest neighbors [51]. Its formula for training 
set is shown as

$$
|\tau|=\frac{1}{\sqrt{N}} \sum_{p}\left|v_{1}^{p} \cdots . v_{n}^{p}, c^{p}\right|
$$

After transforming it in to the quantum state and merging it with the hamming distance, we get,

$$
\left|\varnothing_{n}\right|=\tau\left|\varnothing_{n-1}\right|=\alpha \sum_{p \in \cap}\left|d_{1}^{p} \cdots . d_{n}^{p} ; v_{1}^{p} \cdots . v_{n}^{p}, c^{p} ; 1\right|,
$$

where $\tau$ is the training set, $N$ refers to feature vectors $v^{p}(p$ $=1 \cdots N)$, and the $c^{p}$ is the corresponding class. Another quantum AI computation is the quantum decision tree classifier, which benefits from a preparation dataset including views about facts that are either collected precisely or received from professionals [17]. The preparation dataset is composed of quantum objects rather than perceptions based on conventional knowledge in a quantum environment. A quantum state, $x^{i}$, is spoken to by a $d$-dimensional property vector with its ascribes $a 1, a 2, a 3, a 4 \ldots$ an [49]. The quantum state belongs to space $S_{i}$ and is described as shown in

$$
\varnothing=\sum_{j=1}^{m_{i}} \alpha_{i, j}\left|v_{i, j}\right|
$$

where domain value is represented by $v_{i, j}, \varnothing$ is the quantum state and the coefficients $\left|\boldsymbol{\alpha}_{i, j}\right|^{2}=\mathbf{1}$, and at the end, we have quantum Gaussian Naïve Bayes classifier in which the development of a quantum mechanical description returns the simplest form of Baye's theorem [52] using the case of exclusive populations which is shown as

$$
P\left(H_{1}\right)=\frac{n\left(H_{1}\right)}{n\left(H_{1}\right)+n\left(H_{2}\right)},
$$

where $H$ defines the exclusive population and datasets $D$ as shown in Equation (9).

3.4. Evaluative Parameters. The performance of the proposed system for heart failure detection has been compared on the grounds of accuracy, $F 1$ score, recall, and precision which are described as under $[2,5,53,54]$ :

(i) Accuracy: accuracy is characterized as the level of effectively arranged occurrences which is shown in

$$
\text { Accuracy }=\frac{\mathrm{TP}+\mathrm{TN}}{\mathrm{TP}+\mathrm{TN}+\mathrm{FP}+\mathrm{FN}},
$$

where TP, FN, FP, and TN represent the number of true positives, false negatives, false positives, and true negatives, respectively. To obtain good classifier, the values of TPR and TNR should be nearer to $100 \%$. (ii) F1 score: it is likewise called as $F$ measure. It passes on the harmony between the accuracy and review. It is represented as

$$
F 1 \text { score }=\frac{\mathrm{TP}}{\mathrm{TP}+1 / 2(\mathrm{FP}+\mathrm{FN})},
$$

where $\mathrm{TP}=$ count of true positives, $\mathrm{FP}=$ count of false positives, and $\mathrm{FN}=$ count of false negatives.

(iii) Recall: the recall is the portion of recovered examples among every important occurrence. It is the incentive between 0.0 for no recall and 1.0 for full recall. It is determined as the quantity of genuine positives separated by the all-out number of genuine positives and bogus negatives. It is represented by

$$
\text { Recall }=\frac{\mathrm{TP}}{(\mathrm{TP}+\mathrm{FN})},
$$

where $\mathrm{TP}=$ number of true positives and $\mathrm{FN}=$ number of false negatives.

(iv) Precision: precision is the part of significant cases among the recovered occasions. It is determined as the quantity of genuine positives isolated by the complete number of genuine positives and bogus positives. Equation (17) is represented as:

$$
\text { Precision }=\frac{\mathrm{TP}}{(\mathrm{TP}+\mathrm{FP})},
$$

where TP $=$ count of true positives and FP = count of false positives.

\section{Result and Analysis}

Results are obtained by applying feature transformation and scaling techniques to normalize the data, so that applied machine and quantum-enhanced machine learning models will treat the data equally. The techniques are min-max scalar, standard scalar, and PCA scalar, from where the results are obtained and are later optimized using the pipelining technique. It is further incorporated into the machine and quantum-enhanced machine learning models for heart failure detection. To begin, the min-max scalar technique is used, which is a complement to $Z$-scalar normalization. The data is scaled to a defined range, often 0 to 1 . They are using this method in comparison to standardization; the expense of having this constrained range results in smaller standard deviations and the suppression of outliers. A min-max scaling is performed using

$$
X \mathrm{sc}=X-\frac{X \min }{X \max -X \min } .
$$

Min-max scales the data in which the minimum value 
TABLE 4: Heart failure detection using min-max scalar.

\begin{tabular}{lcccc}
\hline Algorithms & Accuracy & $F 1$ score & Recall & Precision \\
\hline QRFC & $\mathbf{0 . 8 6}$ & $\mathbf{0 . 8 8}$ & $\mathbf{0 . 9 1}$ & $\mathbf{0 . 8 3}$ \\
RFC & 0.83 & 0.85 & 0.88 & 0.80 \\
QKNN & 0.85 & 0.86 & 0.87 & 0.84 \\
KNN & 0.82 & 0.83 & 0.84 & 0.81 \\
QDTC & 0.75 & 0.75 & 0.76 & 0.73 \\
DTC & 0.77 & 0.79 & 0.80 & 0.76 \\
QGNBC & 0.81 & 0.84 & 0.87 & 0.80 \\
GNBC & 0.81 & 0.83 & 0.86 & 0.79 \\
\hline
\end{tabular}

TABLE 5: Heart failure detection using standard scalar.

\begin{tabular}{lcccc}
\hline Algorithms & Accuracy & $F 1$ score & Recall & Precision \\
\hline QRFC & 0.85 & 0.87 & 0.90 & 0.85 \\
RFC & 0.83 & 0.84 & 0.87 & 0.81 \\
QKNN & $\mathbf{0 . 8 8}$ & $\mathbf{0 . 8 9}$ & $\mathbf{0 . 9 0}$ & $\mathbf{0 . 8 7}$ \\
KNN & 0.85 & 0.86 & 0.87 & 0.84 \\
QDTC & 0.75 & 0.76 & 0.76 & 0.73 \\
DTC & 0.77 & 0.79 & 0.80 & 0.76 \\
QGNBC & 0.83 & 0.85 & 0.88 & 0.81 \\
GNBC & 0.81 & 0.83 & 0.86 & 0.79 \\
\hline
\end{tabular}

between the columns became 0 , and the maximum value is changed to 1 with other dataset values in between. After scaling using min-max and obtained, the results for different heart failure detection models have been shown in Table 4 .

Second, apply the standard scalar to the heart failure dataset, resulting in two variables with values ranging from 10 to 100 and 1000 to 5000 . We can compute the biased result by utilizing these predictor values since the variable with the most extensive range will significantly influence the outcome. As a result, it is necessary to normalize the data to a narrow range. Standardization is calculated by subtracting each number from the mean and dividing it by dataset's overall variance. The results of the application of the standard scalar are displayed in Table 5, which was computed using

$$
x_{\text {scaled }}=\text { variable value }-\frac{\text { mean }}{\text { standard deviation }} \text {. }
$$

Then, principal component analysis (PCA), a technique for reducing data dimension, was used to detect the relationships. Additionally, patterns in a dataset may be used to generate a lower-dimensional dataset without sacrificing any information. Thus, PCA analyzes the heart disease dataset to determine the high association between various factors. To preserve the critical data, a final choice is taken to decrease data's massive dimensions. This method is excellent for solving complex data-driven challenges that need the utilization of large datasets. It begins by standardizing the heart disease data, computing the covariance matrix, and calculating the calculated features' eigenvectors and eigenvalues.
TABle 6: Heart failure detection using PCA.

\begin{tabular}{lcccc}
\hline Algorithms & Accuracy & $F 1$ score & Recall & Precision \\
\hline QRFC & 0.82 & 0.84 & 0.90 & 0.79 \\
RFC & 0.79 & 0.81 & 0.87 & 0.76 \\
QKNN & $\mathbf{0 . 8 4}$ & 0.85 & 0.88 & $\mathbf{0 . 8 3}$ \\
KNN & 0.81 & 0.82 & 0.85 & 0.80 \\
QDTC & 0.75 & 0.90 & 0.80 & 0.70 \\
DTC & 0.79 & $\mathbf{0 . 9 2}$ & 0.84 & 0.73 \\
QGNBC & 0.82 & 0.85 & $\mathbf{0 . 9 3}$ & 0.80 \\
GNBC & 0.80 & 0.82 & 0.90 & 0.76 \\
\hline
\end{tabular}

Finally, it adds the main components and decreases the size of the heart disease dataset (see Table 6).

$$
z=\frac{\text { value }- \text { mean }}{\text { standard deviation }},
$$

where standardization equalises the contributions of the many continuous variables to the analysis, subtracting the mean and dividing by the standard deviation for each variable's value, as indicated in Equation (18), maybe done mathematically. After that, the covariance matrix explains the rationale behind the deviation of the input dataset from the mean for each other or the probability of a link. Equation (21) is used to create the matrix.

$$
\operatorname{cov}(X, Y)=\frac{1}{n-1} \sum_{i=1}^{n}\left(X_{i}-\bar{x}\right)\left(Y_{i}-\bar{y}\right)
$$

where $x$ and $y$ are variables; they are positively associated if the two variables rise or decrease simultaneously, but they are negatively connected if one increases while the other drops. To determine the primary components of the data, the third step is to compute the eigenvectors and eigenvalues of the resulting covariance matrix. If $A$ is a square matrix, $v$ is a vector, and $s$ is a scalar, then is the eigenvalue associated with eigenvector $v$ to get the new vectors using

$$
\operatorname{det}(A-\lambda I)=0 \text {. }
$$

Finally, the collected data must be recast along the principal component axis. Its objective is to employ feature vectors derived from the covariance matrix's eigenvectors to reorient the data along the original axis to those represented by principle components, a process referred to as principal component analysis. The results obtained after applying it to the dataset are shown in Table 6.

Finally, pipelines are employed to optimize data to achieve faster outcomes. It automates the machine learning workflow by transforming the data sequence and then correlating them together in a model that can be tested and analyzed to determine if a result is positive or negative. We have performed several steps to train the machine and quantumenhanced machine learning models to optimize the performance using the pipelining technique. It provides flexible 
TABLE 7: Heart failure detection after pipelining.

\begin{tabular}{lcccc}
\hline Algorithms & Accuracy & $F 1$ score & Recall & Precision \\
\hline QRFC & $\mathbf{0 . 8 9}$ & $\mathbf{0 . 8 8}$ & $\mathbf{0 . 9 3}$ & $\mathbf{0 . 8 9}$ \\
RFC & 0.84 & 0.86 & 0.90 & 0.83 \\
QKNN & 0.87 & 0.86 & 0.92 & 0.81 \\
KNN & 0.82 & 0.83 & 0.90 & 0.78 \\
QDTC & 0.81 & 0.80 & 0.78 & 0.85 \\
DTC & 0.80 & 0.79 & 0.75 & 0.84 \\
QGNBC & 0.85 & 0.86 & 0.91 & 0.82 \\
GNBC & 0.84 & 0.85 & 0.90 & 0.81 \\
\hline
\end{tabular}

implementation and performs various tasks such as data collection, cleaning, feature extraction, labelling with dimensionality reduction, and model validation and visualization. The results obtained after applying it to the dataset are shown in Table 7.

On assaying Tables 4, 5, 6, and 7, evaluative parameters such as accuracy, $F 1$ score, recall, and precision that have been obtained using quantum-enhanced and traditional machine learning algorithms are compared based on minmax scalar, standard scalar, principal component analysis, and pipelining optimization as shown in Figure 3.

On applying the min-max scaling technique, the quantum random forest classifier showed the highest accuracy rate by $0.86, F 1$ score by 0.88 , recall value by 0.91 , and precision score by 0.83 as compared to other algorithms. After going through the standard scalar, it can be seen that quantum $K$ nearest neighbor achieved the highest accuracy rate by $0.88, F 1$ score by 0.89 , precision value by 0.87 , and recall value has been shared by quantum random forest classifier as well as quantum $K$ nearest neighbor by 0.9 each. Likewise, when principal component analysis had been applied, quantum $K$ nearest neighbor achieved highest accuracy rate by 0.84 and precision value by 0.83 , decision tree classifier achieved highest $F 1$ score by 0.92 , and quantum Gaussian Naïve Bayes classifier secured highest recall value by 0.93 .

As per the process, these algorithms were further passed through optimization techniques, i.e., pipelining, where the quantum random forest classifier marked the highest values in accuracy, $F 1$ score, recall, and precision by $0.89,0.88$, 0.93 , and 0.89 , respectively. Hence, it can be concluded that quantum-enhanced machine learning algorithms are better than traditional machine learning algorithms in heart failure detection because of their remarkable achievements for all evaluative parameters.

4.1. Comparison with State of the Art Techniques. The comparison shown in Table 8 is based on the processing time taken by the traditional machine learning algorithms and quantum-enhanced machine learning algorithms. For example, the table states that in traditional machine learning algorithms, random forest classifier shows less computation time, i.e., 193 microseconds. In comparison, as $K$ nearest neighbouring classifier shows the worst processing time by 301 microseconds. Likewise, in the quantum-enhanced machine learning algorithm, the quantum random forest classifier executes in 150 microseconds while the quantum decision tree algorithm used 286 microseconds for computation.

Hence, after summing up all these results, including the computational time, it has been observed that after applying the pipelining technique for optimizing the results, quantum random forest stands out to be the best algorithm for detecting heart failure in patients as compared to the other quantum and traditional machine learning algorithms in terms of accuracy, precision, $F 1$ score, recall, and computational time. Table 9 shows the preliminary effects of this work as opposed to state-of-the-art techniques, showing that the proposed work stands out to be better than the state-ofthe-art techniques in all heart failure identification categories.

\section{Future Research Perspective of QML in the Healthcare}

With the advancement in technology, the healthcare sector has gained immense popularity and has significantly benefited [8]. These days, quantum-enhanced machine learning plays a vital role in many health-related sectors, in addition to the development of new medical procedures, maintaining and handling patient data and records, and the therapeutics of chronic diseases. In this tech-savvy world, quantum-enhanced machine learning is popularly used to build rationalized administrative processes in medical institutes to map and treat contagious diseases effectively [35].

Quantum computing possesses an intelligent multitier storage capacity solution that helps in enabling the full power of AI in health and medicine [23]. The storage solution is best suited for video applications and also yields great performance in an organization. The multitier storage framework is more pronounced for its immense benefits in healthcare and medicine. Balanced performance, large capacity, and reduced cost are some of the benefits of building a cost-effective approach for the retention of data [57]. Based on analysis done so far, it can be observed that quantum-enhanced machine learning has tremendous applications in the field of the medical department as shown below:

(i) Medical diagnostics and treatment: quantumenhanced machine learning can be advantageous in the field of health and medicine. This field helps in the easy execution of medical services by diluting costs and enhancing the services to patients for medical treatment and examination [30]

(ii) Heart treatment: nowadays, QML can build pioneering methods to determine the heart rate through magnetic flux and other more robust imaging techniques. In addition to that, quantum computers will be able to analyse and handle data much more efficiently than old conventional computers, which can be used in CT or magnetic resonance imaging [17] 

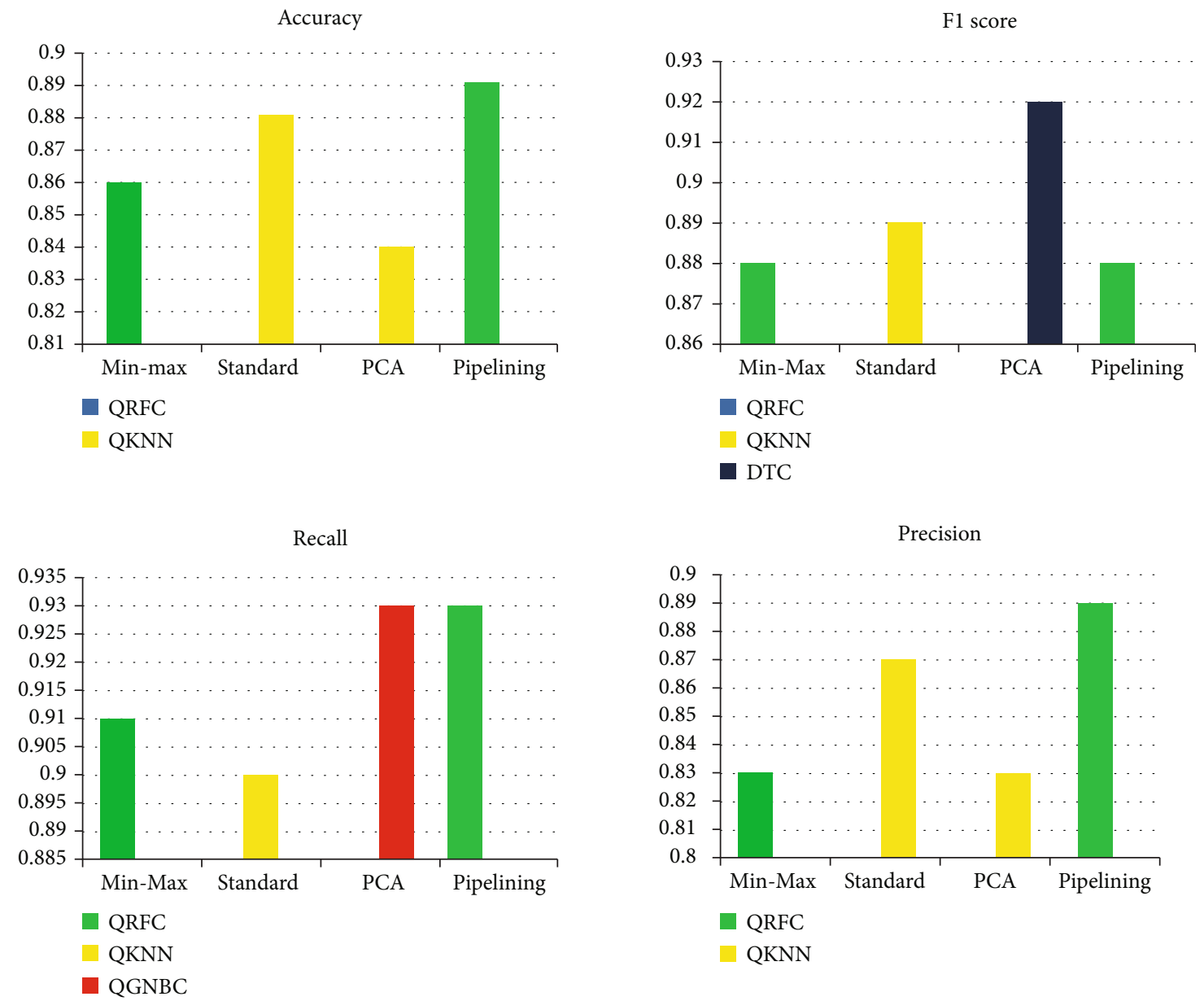

FIGURE 3: Comparison based on evaluative parameters.

TABLE 8: Computational time-based comparative analysis between traditional and quantum-enhanced machine learning algorithms.

\begin{tabular}{lccc}
\hline $\begin{array}{l}\text { Traditional } \\
\text { machine } \\
\text { learning } \\
\text { algorithm }\end{array}$ & $\begin{array}{c}\text { Computation } \\
\text { time }(\mu \mathrm{s})\end{array}$ & $\begin{array}{c}\text { Quantum- } \\
\text { enhanced } \\
\text { machine } \\
\text { learning }\end{array}$ & $\begin{array}{c}\text { Computation } \\
\text { time }(\mu \mathrm{s})\end{array}$ \\
\hline RFC & $\mathbf{1 9 3}$ & QRFC & $\mathbf{1 5 0}$ \\
KNN & 301 & QKNN & 245 \\
DTC & 292 & QDTC & 286 \\
GNBC & 260 & QNGBC & 236 \\
\hline
\end{tabular}

TABle 9: Comparative analysis of proposed method with state-ofart techniques.

\begin{tabular}{lcccc}
\hline State-of-the-art & Accuracy & F1-score & Recall & Precision \\
\hline Dunjiko, V et al. [10] & 0.86 & 0.71 & 0.71 & 0.74 \\
Obiri, D et al. [18] & 0.79 & 0.81 & 0.80 & 0.84 \\
Gao, X et al. [55] & 0.83 & 0.85 & 0.81 & 0.88 \\
Rajdhan, A et al. [56] & 0.85 & 0.86 & 0.85 & 0.88 \\
Proposed work & $\mathbf{0 . 8 9}$ & $\mathbf{0 . 8 8}$ & $\mathbf{0 . 9 3}$ & $\mathbf{0 . 8 9}$ \\
\hline
\end{tabular}

(iii) Cancer detection: cancer research is yet again a different area that is benefitted from the computational power of quantum computing. Clinic experts would be able to treat patients more effectively and timely by enhancing the speed of transferring the data from lab to bedside [58]

(iv) Biomedical imaging: enhanced imaging is a significant process that helps in the premature detection of even minute changes in the body, thereby providing other alternative treatment options for patients affected with diseases such as cancer or dementia, which will improve outcomes. Quantum learning has proved to be a promising method in biomedical imaging [59]

(v) Complex optimization problems: artificial neural network has resulted to be a precise diagnostic approach in traditional machine learning, which is optimized by varying the specifications of network's framework. These methods of optimization are convenient for quantum computing, where the propensity of "quantum tunnelling" fosters optimization problems to be computed quickly $[11,12,18,34,35]$

The two most important applications are quantumenhanced sampling and discrete optimization. Quantum- 
enhanced sampling is the process of extracting a slice of a probability distribution from a quantum system, and in finance, discrete optimization is used to maximize the yield of a group of financial properties, which is an optimization challenge, where as in most cases, shallow learning approaches are inaccurate. In addition to this, the study of medical images, logistics, scheduling, climate modeling, weather forecasting, cryptography, and artificial intelligence may benefit from quantum-enhanced machine learning. These use cases collectively contribute significantly to the quadruple goal of healthcare.

Although quantum-enhanced machine learning enhances computing speed and can manage data storage utilizing various techniques, its limits are still readily apparent in practice. The study is confined to a minimally viable solution model. More research in heart disease detection may be required to convert the current model into a quickly deployable services mode, allowing for large-scale use in clinical trials. It also faces several hardware and software challenges [34], including quantum decoherence caused by heat and light, which causes qubits to lose their quantum properties such as entanglement, which results in the loss of stored data. Rotations in quantum computers' logic gates are prone to generate inaccuracy, as any incorrect rotation can result in an error in the output. Likewise, quantum algorithms face the constraint of specific simulations that limit their application.

Hence, the suggested quantum-enhanced machine learning model will need to be integrated with a deep learning framework in the future to improve its performance on comparing with the previously built models and state-of-the-art methodologies.

\section{Conclusion}

An empirical study has been made with regard to the diverse range of applications of quantum-enhanced machine learning in healthcare. Several authors shared the same idea of using traditional machine learning algorithms to compute a substantial amount of data to compute data intelligently. The primary objective of this study is to have a comparative analysis of standard and quantum-enhanced machine learning algorithms for the prediction of heart failure illness. In the previous work, researchers have used traditional machine learning algorithms in the health care sector, which lacked in terms of accuracy, computation time, and performance as compared to quantum-enhanced machine learning algorithms as they speed up the processing of information, show higher accuracy rate, and also, increase the performance of the system. Thus, in this article, we have performed the experiment by considering various traditional machine learning algorithms and quantum-enhanced machine learning algorithms to make the comparison to depict the best algorithm for heart failure detection. The detailed analysis of the techniques shows that quantumenhanced machine learning significantly contributes to medical science and healthcare.

We have also shown that the prediction of heart failure as the cause of death can be effectively and more precisely be predicted with the help of QML algorithms. For that purpose, we have performed the scaling and transformation of the results by applying the scaling methods and then optimized it by using pipelining. We have seen a considerable increment in all the performance metrics like accuracy, $F 1$ -score, precision, and recall. The large datasets are handled much better by quantum computing, which is its primary objective. Heart rates, temperature, blood pressure, oxygen levels, and other parameters will be monitored by quantum-enhanced machine learning algorithms with the internet of medical things, including smartwatches, fitness wearables, and smartphones. Patients' vital signs, such as blood pressure, heart rate, and electrocardiography (ECG), will be collected and transmitted to a healthcare practitioner using IoMT devices, providing a quick and accurate picture of patient's state of health and well-being. Additionally, the substantial analysis of quantum-enhanced machine learning tools on the diseases and abnormalities soaring rapidly supports a strong candidature in controlling these diseases at an early stage.

\section{Data Availability}

The data used to support the findings of this study are available upon request.

\section{Conflicts of Interest}

The authors declare no conflict of interest.

\section{Acknowledgments}

Jana Shafi would like to thank the Deanship of Scientific Research, Prince Sattam bin Abdul Aziz University for supporting this work.

\section{References}

[1] M. Amin-Naji, H. Mahdavinataj, and A. Aghagolzadeh, “Alzheimers disease diagnosis from structural MRI using Siamese convolutional neural network," in 2019 4th International Conference on Pattern Recognition and Image Analysis (IPRIA), pp. 75-79, Tehran, Iran, 2019.

[2] J. Luyapan, X. Ji, D. Zhu, T. Mackenzie, I. Amos, and J. Gui, "An efficient survival multifactor dimensionality reduction method for detecting gene-gene interactions of lung cancer onset age," in 2018 IEEE International Conference on Bioinformatics and Biomedicine (BIBM), pp. 2779-2781, Madrid, Spain, 2018.

[3] P. Rani, S. V. Kavita, S. Verma, and G. N. Nguyen, "Mitigation of black hole and gray hole attack using swarm inspired algorithm with artificial neural network," Access, vol. 8, pp. 121755-121764, 2020.

[4] S. Ramisetty, S. Varma, and S. Varma, "The amalgamative sharp wireless sensor networks routing and with enhanced machine learning," Journal of Computational and Theoretical Nanoscience, vol. 16, no. 9, pp. 3766-3769, 2019.

[5] A. Gonsalves, F. Thabtah, R. Mohammad, and G. Singh, "Prediction of coronary heart disease using machine learning: an experimental analysis," in Proceedings of the 2019 3rd 
International Conference on Deep Learning Technologies, pp. 51-56, Xiamen, China, 2019.

[6] W. Hu, "Comparison of two quantum nearest neighbor classifiers on IBM's quantum simulator," Natural Science, vol. 10, no. 3, pp. 87-98, 2018.

[7] J. Bang, S. W. Lee, and H. Jeong, "Protocol for secure quantum machine learning at a distant place," Quantum Information Processing, vol. 14, no. 10, pp. 3933-3947, 2015.

[8] X. Tian, Y. Huang, S. Verma et al., "Power allocation scheme for maximizing spectral efficiency and energy efficiency tradeoff for uplink NOMA systems in B5G/6G," Physical Communication, vol. 43, 2020.

[9] M. Abdel-Basset, A. Gamal, G. Manogaran, L. Son, and V. H. Long, "A novel group decision making model based on neutrosophic sets for heart disease diagnosis," Multimedia Tools and Applications, vol. 79, no. 15-16, pp. 9977-10002, 2020.

[10] K. Wereszczynski, A. Michalczuk, H. Josinski, and A. Polanski, "Quantum computing for clustering big datasets," in 2018 Applications of Electromagnetics in Modern Techniques and Medicine (PTZE), pp. 276-280, Racławice, Poland, 2018.

[11] J. A. Alzubi, A. Yaghoubi, M. Gheisari, and Y. Qin, "Improve heteroscedastic discriminant analysis by using CBP algorithm," in Algorithms and Architectures for Parallel Processing, J. Vaidya and J. Li, Eds., vol. 11335 of Lecture Notes in Computer Science, , Springer, 2018.

[12] M. Gheisari, "The effectiveness of schema therapy integrated with neurological rehabilitation methods to improve executive functions in patients with chronic depression," Health Science Journal, vol. 10, 2016.

[13] M. Kumar, P. Mukherjee, K. Verma, S. Verma, and D. B. Rawat, "Improved deep convolutional neural network based malicious node detection and energy-efficient data transmission in wireless sensor networks," IEEE Transactions on Network Science and Engineering, vol. 25, 2021.

[14] G. Rani, G. Oza, S. Dhaka, N. Pradhan, S. Verma, and J. J. P. C. Rodrigues, "Applying deep learning-based multi-modal for detection of coronavirus," Multimedia Systems, vol. 27, 12 pages, 2021.

[15] Z. Lv, L. Qiao, and S. Verma, "AI-enabled IoT-edge data analytics for connected living," ACM Transactions on Internet Technology, vol. 21, pp. 1-20, 2021.

[16] M. Arora, S. Verma, C. S. Kavita, and S. Chopra, "A systematic literature review of machine learning estimation approaches in scrum projects," in Cognitive Informatics and Soft Computing, P. Mallick, V. Balas, A. Bhoi, and G. S. Chae, Eds., vol. 1040 of Advances in Intelligent Systems and Computing, pp. 573-586, Springer, Singapore, 2020.

[17] X. Gao, A. Amin Ali, H. Shaban Hassan, and M. Anwar, "Improving the accuracy for analyzing heart diseases prediction based on the ensemble method," Complexity, vol. 2021, Article ID 6663455, 10 pages, 2021.

[18] M. Rathi and A. Gupta, "Mobile-based prediction framework for disease detection using hybrid data mining approach," Proceedings of International Conference on Artificial Intelligence and Applications, , pp. 521-530, Springer, 2021.

[19] Z. Li, S. Verma, and M. Jin, "Power allocation in massive MIMO-HWSN based on the water-filling algorithm," Wireless Communications and Mobile Computing, vol. 2021, Article ID 8719066, 11 pages, 2021.

[20] F. Ablayev, M. Ablayev, J. Huang, K. Khadiev, N. Salikhova, and $\mathrm{D}$. $\mathrm{Wu}$, "On quantum methods for machine learning problems part II: quantum classification algorithms," Big Data Mining and Analytics, vol. 3, no. 1, pp. 56-67, 2020.

[21] Y. Kumar, "Recent advancement of machine learning and deep learning in the field of healthcare system," in Computational Intelligence for Machine Learning and Healthcare Informatics, R. Srivastava, P. K. Mallick, S. S. Rautaray, and M. Pandey, Eds., pp. 7-98, De Gruyter, Berlin, Boston, 2020.

[22] S. Qaisar and A. Subasi, "Cloud-based ECG monitoring using event-driven ECG acquisition and machine learning techniques," Physical and Engineering Sciences in Medicine, vol. 43, no. 2, pp. 623-634, 2020.

[23] C. Chen and D. Dong, "Superposition-inspired reinforcement learning and quantum reinforcement learning," in Reinforcement Learning, pp. 1-5, IntechOpen, 2008.

[24] L. Gaur, G. Singh, A. Solanki et al., "Disposition of youth in predicting sustainable development goals using the neurofuzzy and random forest algorithms," Human-Centric Computing and Information Sciences, vol. 11, p. 24, 2021.

[25] Y. Dang, N. Jiang, H. Hu, Z. Ji, and W. Zhang, "Image classification based on quantum K-nearest-neighbor algorithm," Quantum Information Processing, vol. 17, no. 9, 2018.

[26] S. Lu and S. Braunstein, "Quantum decision tree classifier," Quantum Information Processing, vol. 13, no. 3, pp. 757-770, 2014.

[27] N. Bharill, O. Patel, and A. Tiwari, "An enhanced quantuminspired evolutionary fuzzy clustering," in 2015 IEEE Symposium Series on Computational Intelligence, pp. 772-779, Cape Town, South Africa, 2015.

[28] H. Wang, J. Liu, J. Zhi, and C. Fu, "The improvement of quantum genetic algorithm and its application on function optimization," Mathematical Problems in Engineering, vol. 2013, Article ID 730749, 10 pages, 2013.

[29] S. Taha and Z. Taha, "EEG signals classification based on autoregressive and inherently quantum recurrent neural network," International Journal of Computer Applications in Technology, vol. 58, no. 4, pp. 340-351, 2018.

[30] I. Wallach, M. Dzamba, and A. Heifets, "AtomNet: a deep convolutional neural network for bioactivity prediction in structure-based drug discovery," http://arxiv.org/abs/1510 $.02855 \mathrm{v} 1$.

[31] D. Sierra-Sosa, J. Arcila-Moreno, B. Garcia-Zapirain, C. Castillo-Olea, and A. Elmaghraby, "Dementia prediction applying variational quantum classifier," http://arxiv.org/ abs/2007.08653.

[32] J. Amin, M. Sharif, N. Gul, S. Kadry, and C. Chakraborty, "Quantum machine learning architecture for COVID-19 classification based on synthetic data generation using conditional adversarial neural network," Cognitive Computation, vol. 13, pp. 1-12, 2021.

[33] H. Gupta, H. Varshney, K. Sharma, N. Pachauri, and P. Verma, "Comparative performance analysis of quantum machine learning with deep learning for diabetes prediction," Complex \& Intelligent Systems, vol. 7, 2021.

[34] M. Gheisari and M. Esnaashari, "A survey to face recognition algorithms: advantageous and disadvantageous," Journal Modern Technology \& Engineering, vol. 2, no. 1, pp. 57-65, 2017.

[35] M. Ashourian, M. Gheisar, and A. H. Talkhoncheh, "An improved node scheduling scheme for resilient packet ring network," Majlesi Journal of Electrical Engineering, vol. 9, no. 2 , p. $43,2015$. 
[36] G. Yang, M. A. Jan, A. U. Rehman, M. Babar, M. M. Aimal, and S. Verma, "Interoperability and data storage in internet of multimedia things: investigating current trends, research challenges and future directions," IEEE Access, vol. 8, pp. 124382-124401, 2020.

[37] G. Ghosh, G. Kavita, D. Anand et al., "Secure surveillance systems using partial-regeneration-based non-dominated optimization and 5D-chaotic map," Symmetry, vol. 13, no. 8, p. 1447, 2021.

[38] S. Verma and S. Mittal, "Implementation and analysis of stability improvement in VANET using different scenarios," International Journal of Engineering \& Technology, vol. 7, pp. 151-154, 2018.

[39] D. F. Parsons, "Possible medical and biomedical uses of quantum computing," Neuroquantology, vol. 9, no. 3, pp. 596-600, 2011.

[40] M. Niemiec, "Error correction in quantum cryptography based on artificial neural networks," Quantum Information Processing, vol. 18, no. 6, pp. 1-18, 2019.

[41] J. Suo, L. Wang, S. Yang, W. Zheng, and J. Zhang, "Quantum algorithms for typical hard problems: a perspective of cryptanalysis," Quantum Information Processing, vol. 19, no. 6, pp. 1-26, 2020.

[42] Y. Kumar, K. Sood, S. Kaul, and R. Vasuja, "Big data analytics and its benefits in healthcare," in Big Data Analytics in Healthcare, A. Kulkarni, Ed., vol. 66 of Studies in Big Data, , pp. 3-21, Springer, 2020.

[43] A. Joseph, T. Hijal, J. Kildea, L. Hendren, and D. Herrera, "Predicting waiting times in radiation oncology using machine learning," in 2017 16th IEEE International Conference on Machine Learning and Applications (ICMLA), pp. 10241029, Cancun, Mexico, 2017.

[44] M. Ijaz, M. Attique, and Y. Son, "Data-driven cervical cancer prediction model with outlier detection and over-sampling methods," Sensors, vol. 20, no. 10, p. 2809, 2020.

[45] M. F. Ijaz, G. Alfian, M. Syafrudin, and J. Rhee, "Hybrid prediction model for type 2 diabetes and hypertension using DBSCAN-based outlier detection, synthetic minority over sampling technique (SMOTE), and random forest," Applied Sciences, vol. 8, no. 8, p. 1325, 2018.

[46] A. Gandam, J. S. Sidhu, S. Verma et al., "An efficient postprocessing adaptive filtering technique to rectifying the flickering effects," PLoS One, vol. 16, no. 5, 2021.

[47] W. Li, Y. Chai, F. Khan et al., "A comprehensive survey on machine learning-based big data analytics for IoT-enabled smart healthcare system," Mobile Network and Applications, vol. 26, no. 1, pp. 234-252, 2021.

[48] J. Bird, A. Ekárt, and D. Faria, "On the effects of pseudorandom and quantum-random number generators in soft computing," Soft Computing, vol. 24, no. 12, pp. 9243-9256, 2020.

[49] M. Hammad, A. M. Iliyasu, A. Subasi, E. Ho, and A. A. A. elLatif, "A multitier deep learning model for arrhythmia detection," IEEE Transactions on Instrumentation and Measurement, vol. 70, pp. 1-9, 2021.

[50] S. Sharma, "Qeml (quantum enhanced machine learning): Using quantum computing to enhance $\mathrm{ml}$ classifiers and feature spaces," vol. 1, 2020, http://arxiv.org/abs/2002.10453.

[51] P. Sharma, K. Choudhary, K. Gupta, R. Chawla, D. Gupta, and A. Sharma, "Artificial plant optimization algorithm to detect heart rate \& presence of heart disease using machine learning,"
Artificial Intelligence in Medicine, vol. 102, pp. 101752101759, 2020.

[52] M. Sood, S. Verma, V. K. Panchal, and Kavita, "Optimal path planning using swarm intelligence based hybrid techniques," Journal of Computational and Theoretical Nanoscience, vol. 16, no. 9, pp. 3717-3727, 2019.

[53] N. Kundu, G. Rani, V. S. Dhaka et al., "IoT and interpretable machine learning based framework for disease prediction in pearl millet," Sensors, vol. 21, no. 16, p. 5386, 2021.

[54] T. Reddy, S. Bhattacharya, P. K. R. Maddikunta et al., "Antlion re-sampling based deep neural network model for classification of imbalanced multimodal stroke dataset," Multimedia Tools and Applications, vol. 80, pp. 1-25, 2020.

[55] S. Qaisar and F. Hussain, “An effective arrhythmia classification via ECG signal subsampling and mutual information based subbands statistical features selection," Journal of Ambient Intelligence and Humanized Computing, vol. 12, 2021.

[56] A. Rajdhan, A. Agarwal, M. Sai, D. Ravi, and P. Ghuli, "Heart disease prediction using machine learning," International Journal of Engineering Research and Technology, vol. 9, no. 4, 2020.

[57] S. Gupta, S. Mohanta, M. Chakraborty, and S. Ghosh, "Quantum enhanced machine learning-using quantum computation in artificial intelligence and deep neural networks: quantum computation and machine learning in artificial intelligence," in 2017 8th Annual Industrial Automation and Electromechanical Engineering Conference (IEMECON), pp. 268-274, Bangkok, Thailand, 2017.

[58] G. T. Reddy, M. P. K. Reddy, K. Lakshmanna, D. S. Rajput, R. Kaluri, and G. Srivastava, "Hybrid genetic algorithm and a fuzzy logic classifier for heart disease diagnosis," Evolutionary Intelligence, vol. 13, no. 2, pp. 185-196, 2020.

[59] M. Willsch, D. Willsch, F. Jin, H. Raedt, and K. Michielsen, "Benchmarking the quantum approximate optimization algorithm," Quantum Information Processing, vol. 19, no. 7, pp. 1-24, 2020. 